Bangladesh J. Plant Taxon. 19(1): 55-61, 2012 (June)

(C) 2012 Bangladesh Association of Plant Taxonomists

\title{
FIFTY NEW COMBINATIONS IN PHANERA LOUR. (LEGUMINOSAE: CAESALPINIOIDEAE) FROM PALEOTROPICAL REGION
}

\author{
S. BANDYOPADHYAY ${ }^{1}$, P.P. GHOSHAL AND M.K. PATHAK \\ Central National Herbarium, Botanical Survey of India, P.O. Botanic Garden, \\ Howrah 711 103, West Bengal, India \\ Keywords: Leguminosae; Caesalpinioideae; Bauhinia; Phanera; New combinations.
}

\begin{abstract}
Fifty new combinations are proposed in Phanera for species earlier treated in Bauhinia L.

\section{Introduction}

The subgenus Phanera (Lour.) Kurz (Wunderlin et al., 1987; Bandyopadhyay, 1999) under Bauhinia L. is now recognized as a genus distinct from Bauhinia based on recent advances in molecular phylogeny (Lewis and Forest, 2005; Bruneau et al., 2008; Sinou et al., 2009). Thirty two new combinations have already been proposed in Phanera Lour. occurring in the Neotropical region from Brazil (Queiroz, 2006; Vaz, 2010) and for one species from the Palaeotropical region (Wunderlin, 2011). Fifty new combinations are proposed here in Phanera occurring in the Palaeotropical region from China, India and Myanmar to Malesia along with the types of the names which have been cited either by scrutinizing the relevant protologues or from the publication of Larsen and Larsen (1996).
\end{abstract}

1. Phanera aherniana (Perkins) de Wit var. subglabra (Merr.) Bandyop., P.P. Ghoshal et M.K. Pathak, comb. et stat. nov.

Bauhinia subglabra Merr., Philipp. J. Sci., C. 3: 230 (1908).

Bauhinia aherniana Perkins var. subglabra (Merr.) K. Larsen \& S.S. Larsen, Nordic J. Bot. 13: 661 (1993). Type: F.W. Foxworthy, Bureau of Science no. 821 (K, image! lecto; NY, image! isolecto).

2. Phanera andersonii (K. Larsen \& S.S. Larsen) Bandyop., P.P. Ghoshal et M.K. Pathak, comb. nov.

Bauhinia andersonii K. Larsen \& S.S. Larsen, Nordic J. Bot. 2: 330 (1982). Type: UNESCO Limestone Expedition 1962, no. 653 (K, image! holo; L, SING, iso).

3. Phanera aureifolia (K. Larsen \& S.S. Larsen) Bandyop., P.P. Ghoshal et M.K. Pathak, comb. nov.

Bauhinia aureifolia K. Larsen \& S.S. Larsen, Nordic J. Bot. 11: 633 (1991).

Bauhinia chrysophylla K. Larsen \& S.S. Larsen, Nordic J. Bot. 9: 253 (1989), non Vahl ex DC. (1825) nec Vogel (1839). Type: C. Niyomdham \& W. Ueachirakan 1837 (AAU, holo; $\mathrm{BKF}, \mathrm{K}$, image! iso).

4. Phanera bidentata (Jack) Benth. var. breviflora (Ridl.) Bandyop., P.P. Ghoshal et M.K. Pathak, comb. et stat. nov.

Bauhinia breviflora Ridl., Fl. Malay Penins. 5: 306 (1925).

Bauhinia bidentata Jack var. breviflora (Ridl.) K. Larsen \& S.S. Larsen, Nordic J. Bot. 13: 663 (1993). Type: Pahang, I.H. Burkill \& Haniff, Singapore Field no. 16867 (K, image! holo; SING, iso).

${ }^{1}$ Email: subirbandyopadhyay@yahoo.com 
5. Phanera bidentata (Jack) Benth. var. cornifolia (Baker) Bandyop., P.P. Ghoshal et M.K. Pathak, comb. et stat. nov.

Bauhinia cornifolia Baker in Hook.f., Fl. Brit. India 2: 278 (1878).

Bauhinia bidentata Jack var. cornifolia (Baker) Bennet, Indian J. Forest. 5: 326 (1982).

Type: Griffith 1878 (K, image!).

6. Phanera bidentata (Jack) Benth. var. gracilipes (Merr.) Bandyop., P.P. Ghoshal et M.K. Pathak, comb. et stat. nov.

Bauhinia gracilipes Merr., Pap. Michigan Acad. Sci. 19: 157 (1934).

Bauhinia bidentata Jack var. gracilipes (Merr.) K. Larsen \& S.S. Larsen, Nordic J. Bot. 13: 663 (1993). Type: H.H. Bartlett 7600 (A, holo; C, L, image!, NY, image!, W, iso).

7. Phanera bidentata (Jack) Benth. var. monticola (Ridl.) Bandyop., P.P. Ghoshal et M.K. Pathak, comb. et stat. nov.

Bauhinia monticola Ridl., J. Straits Branch Roy. Asiat. Soc. 75: 28 (1917).

Bauhinia bidentata Jack var. monticola (Ridl.) K. Larsen \& S.S. Larsen, Nordic J. Bot. 13: 663 (1993). Type: H.C. Robinson s.n. (SING, holo; K, image! iso).

8. Phanera bracteata Benth. subsp. astylosa (K. Larsen \& S.S. Larsen) Bandyop., P.P. Ghoshal et M.K. Pathak, comb. nov.

Bauhinia bracteata (Benth.) J. Graham ex Baker subsp. astylosa K. Larsen \& S.S. Larsen, Fl. Cambodge, Laos \& Vietnam 18: 178 (1980). Type: Dournes s.n. (P, image!).

9. Phanera burbidgei (Stapf) Bandyop., P.P. Ghoshal et M.K. Pathak, comb. nov.

Bauhinia burbidgei Stapf, Trans. Linn. Soc. London, Bot. 4: 143 (1894). Type: O. Beccari 633 (FI, image! holo).

10. Phanera campanulata (S.S. Larsen) Bandyop., P.P. Ghoshal et M.K. Pathak, comb. nov. Bauhinia campanulata S. S. Larsen, Nordic J. Bot. 14: 289 (1994). Type: D.A. Simpson \& M. Marsh 2051 (K, image! holo; AAU, BRUN, iso).

11. Phanera clemensiorum (Merr.) Bandyop., P.P. Ghoshal et M.K. Pathak, comb. nov. Bauhinia clemensiorum Merr., J. Arnold Arbor. 23:171 (1942). Type: J. Clemens \& M.S. Clemens 3621 (A, holo; P, K, image! iso).

12. Phanera excurrens (Stapf) Bandyop., P.P. Ghoshal et M.K. Pathak, comb. nov.

Bauhinia excurrens Stapf, Trans. Linn. Soc. London, Bot. 4: 143 (1894). Type: G.D. Haviland 1382 (K, image! holo).

13. Phanera fabrilis (de Wit) Bandyop., P.P. Ghoshal et M.K. Pathak, comb. et stat. nov. Phanera riedelii (Baker) de Wit var. fabrilis de Wit, Reinwardtia 3: 464 (1956).

Bauhinia fabrilis (de Wit) K. Larsen \& S.S. Larsen, Nordic J. Bot. 11: 634 (1991).

Type: Anthony, B.N.B. Forestry Department, No. A. 756 (L, holo; K, image!, KEP, SING, iso).

14. Phanera foraminifera (Gagnep.) de Wit var. falcata (K. Larsen \& S.S. Larsen) Bandyop., P. P. Ghoshal et M.K. Pathak, comb. nov.

Bauhinia foraminifera Gagnep. var. falcata K. Larsen \& S.S. Larsen, Nordic J. Bot. 13: 661 (1993). Type: P.S. Ashton, BRUN 5614 (L, holo; K, image! iso). 
15. Phanera ferruginea (Roxb.) Benth. var. griffithiana (Benth.) Bandyop., P.P. Ghoshal et M.K. Pathak, comb. et stat. nov.

Phanera griffithiana Benth. in Miq., Pl. Jungh.: 263 (1852).

Bauhinia ferruginea Roxb. var. griffithiana (Benth.) Baker in Hook.f., Fl. Brit. India 2: 283 (1878). Type: Griffith s.n. (K, image!, BR ).

16. Phanera franckii (K. Larsen \& S.S. Larsen) Bandyop., P.P. Ghoshal et M.K. Pathak, comb. nov.

Bauhinia franckii K. Larsen \& S.S. Larsen, Nordic J. Bot. 11: 633 (1991). Type: C.W. Franck 1406 (C, image! holo).

17. Phanera havilandii (Merr.) Bandyop., P.P. Ghoshal et M.K. Pathak, comb. nov. Bauhinia havilandii Merr., Philipp. J. Sci., C. 11: 79 (1916). Type: Native collector, Bureau of Science no. 199 (US, lecto).

18. Phanera khasiana (Baker) Thoth. subsp. polystachya (Gagnep.) Bandyop., P.P. Ghoshal et M.K. Pathak, comb. et stat. nov.

Bauhinia polystachya Gagnep., Notul. Syst. (Paris) 2: 178 (1912).

Bauhinia khasiana Baker subsp. polystachya (Gagnep.) K. Larsen \& S.S. Larsen, Fl. Cambodge, Laos \& Vietnam 18: 189 (1980). Type: Massie s.n. (P, image!).

19. Phanera khasiana (Baker) Thoth. var. gigalobia (D.X. Zhang) Bandyop., P.P. Ghoshal et M.K. Pathak, comb. nov.

Bauhinia khasiana Baker var. gigalobia D.X. Zhang, Nordic J. Bot. 13: 401 (1993).

Type: Cai Kehua 584 (KUN, holo \& iso).

20. Phanera khasiana (Baker) Thoth. var. tomentella (T.C. Chen) Bandyop., P.P. Ghoshal et M.K. Pathak, comb. nov.

Bauhinia khasiana Baker var. tomentella T.C.Chen, Guihaia 8: 46 (1988). Type: W.S. Lious 303 (KUN, holo).

21. Phanera kingii (Prain ex King) Bandyop., P.P. Ghoshal et M.K. Pathak, comb. nov. Bauhinia kingii Prain ex King, J. Asiat. Soc. Bengal, Pt. 2, Nat. Hist. 66: 189 (1897). Type: Scortechini 320 (K, image! lecto).

22. Phanera kockiana (Korth.) Benth. var. angustifolia (K. Larsen \& S.S. Larsen) Bandyop., P.P. Ghoshal et M.K. Pathak, comb. nov.

Bauhinia kockiana Korth. var. angustifolia K. Larsen \& S.S. Larsen, Nordic J. Bot. 13: 664 (1993). Type: W.L. Chew et al. 2968 (K, image! holo; L, image!, SING, iso).

23. Phanera kockiana (Korth.) Benth. var. bakoensis (K. Larsen \& S.S. Larsen) Bandyop., P.P. Ghoshal et M.K. Pathak, comb. nov.

Bauhinia kockiana Korth. var. bakoensis K. Larsen \& S.S. Larsen, Nordic J. Bot. 13: 664 (1993). Type: Ilias Paie S.17902 (L, image! holo; K, SAN, SING, iso).

24. Phanera kockiana (Korth.) Benth. var. beccarii (K. Larsen \& S.S. Larsen) Bandyop., P.P. Ghoshal et M.K.Pathak, comb. nov.

Bauhinia kockiana Korth. var. beccarii K. Larsen \& S.S. Larsen, Nordic J. Bot. 13: 664 (1993). Type: Petrus \& Dewol, SAN 89576 (SAN, holo; AAU, MO, SING, iso). 
25. Phanera kockiana (Korth.) Benth. var. brevipedicellata (K. Larsen \& S.S. Larsen) Bandyop., P.P. Ghoshal et M.K. Pathak, comb. nov.

Bauhinia kockiana Korth. var. brevipedicellata K. Larsen \& S.S. Larsen, Nordic J. Bot. 13: 664 (1993). Type: Mogea 3508 (L, image! holo; BO, iso).

26. Phanera kockiana (Korth.) Benth. var. calcicola (K. Larsen \& S.S. Larsen) Bandyop., P.P. Ghoshal et M.K. Pathak, comb. nov.

Bauhinia kockiana Korth. var. calcicola K. Larsen \& S.S. Larsen, Nordic J. Bot. 13: 664 (1993). Type: P.J. Martin S. 38676 (L, image! holo; AAU, K, KEP, iso).

27. Phanera kockiana (Korth.) Benth. var. scarlatina (Backer ex Cammerl.) Bandyop., P.P. Ghoshal et M.K. Pathak, comb. et stat. nov.

Bauhinia scarlatina Backer ex Cammerl., Bul. Fac. Sti. Cernăuti 3: 171 (1929).

Bauhinia kockiana Korth. var. scarlatina (Backer ex Cammerl.) K. Larsen \& S.S. Larsen, Nordic J. Bot. 13: 665 (1993). Type: Jaheri 1181 (BO, holo; L, image! iso).

28. Phanera kostermansii (K. Larsen \& S.S. Larsen) Bandyop., P.P. Ghoshal et M.K. Pathak, comb. nov.

Bauhinia kostermansii K. Larsen \& S.S. Larsen, Nordic J. Bot. 11: 629 (1991).

Type: A. Kostermans 13549 (L, holo; BO, K, image!, P, iso).

29. Phanera lingua (DC.) Miq. var. antipolana (Perkins) Bandyop., P.P. Ghoshal et M.K. Pathak, comb. et stat. nov.

Bauhinia antipolana Perkins, Fragm. Fl. Philipp. 1: 9 (1904).

Bauhinia lingua DC. var. antipolana (Perkins) K. Larsen \& S.S. Larsen, Nordic J. Bot. 13: 660 (1993). Type: E.D. Merrill 1317 (NY, image! lecto).

30. Phanera lingua (DC.) Miq. var. riedelii (Baker) Bandyop., P.P. Ghoshal et M.K. Pathak, comb. et stat. nov.

Bauhinia riedelii Baker, J. Linn. Soc., Bot. 15: 98 (1876).

Bauhinia lingua DC. var. riedelii (Baker) K. Larsen \& S.S. Larsen, Nordic J. Bot. 13: 660 (1993). Type: Riedel s.n. (K, lecto; P, W, iso).

31. Phanera merrilliana (Perkins) de Wit var. borneensis (K. Larsen \& S.S. Larsen) Bandyop., P.P. Ghoshal et M.K. Pathak, comb. nov.

Bauhinia merrilliana Perkins var. borneensis K. Larsen \& S.S. Larsen, Nordic J. Bot. 13: 658 (1993). Type: Ilias bin Paie S. 28594 (L, image! holo; E, K, SAR, SING, iso).

32. Phanera ornata (Kurz) Thoth. subsp. mizoramensis (Bandyop. et al.) Bandyop., P.P. Ghoshal et M.K. Pathak, comb. nov.

Bauhinia ornata Kurz subsp. mizoramensis Bandyop. et al., Nordic J. Bot. 12: 223 (1992). Type: R. Dutta 33793 (CAL!, holo).

33. Phanera ornata (Kurz) Thoth. var. balansae (Gagnep.) Bandyop., P.P. Ghoshal et M.K. Pathak, comb. et stat. nov.

Bauhinia balansae Gagnep., Notul. Syst. (Paris) 2: 168 (1912).

Bauhinia ornata Kurz var. balansae (Gagnep.) K. Larsen \& S.S. Larsen, Fl. Cambodge, Laos \& Vietnam 18: 209 (1980). Type: M. Balansa 2140 (P, image!). 
34. Phanera ornata (Kurz) Thoth. var. burmanica (K.Larsen \& S.S.Larsen) Bandyop., P.P. Ghoshal et M.K. Pathak, comb. nov.

Bauhinia ornata Kurz var. burmanica K. Larsen \& S.S. Larsen, Thai Forest Bull., Bot. 13: 42 (1980). Type: J. Keenan et al. 735 (E, holo; A, K, image! iso).

35. Phanera ornata (Kurz) Thoth. var. kerrii (Gagnep.) Bandyop., P.P. Ghoshal et M.K. Pathak, comb. et stat. nov.

Bauhinia kerrii Gagnep., Notul. Syst. (Paris) 2: 173 (1912).

Bauhinia ornata Kurz var. kerrii (Gagnep.) K. Larsen \& S.S. Larsen, Fl. Cambodge, Laos \& Vietnam 18: 208 (1980). Type: Kerr 1740 (K, image! lecto; P, image!, BM, image! isolecto).

36. Phanera ornata (Kurz) Thoth. var. subumbellata (Pierre ex Gagnep.) Bandyop., P.P. Ghoshal et M.K. Pathak, comb. et stat. nov.

Bauhinia subumbellata Pierre ex Gagnep., Notul. Syst. (Paris) 2: 180 (1912).

Bauhinia ornata Kurz var. subumbellata (Pierre ex Gagnep.) K. Larsen \& S.S. Larsen, Fl. Cambodge, Laos \& Vietnam 18: 209 (1980). Type: Harmand 1119 (P, image! holo).

37. Phanera pachyphylla (Merr.) de Wit var. wenzelii (K. Larsen \& S.S. Larsen) Bandyop., P.P. Ghoshal et M.K. Pathak, comb. nov.

Bauhinia pachyphylla Merr. var. wenzelii K. Larsen \& S.S. Larsen, Nordic J. Bot. 13: 659 (1993). Type: Wenzel 2537 (NY, image! holo; BO, BR, K, MO, PR, iso).

38. Phanera rahmatii (Merr.) Bandyop., P.P. Ghoshal et M.K. Pathak, comb. nov.

Bauhinia rahmatii Merr., Pap. Michigan Acad. Sci. 19: 158 (1934). Type: Rahmat Si Toroes 161 (A, holo; NY, image! iso).

39. Phanera ridleyi (Prain ex King) Bandyop., P.P. Ghoshal et M.K. Pathak, comb. nov. Bauhinia ridleyi Prain ex King, J. Asiat. Soc. Bengal, Pt. 2, Nat. Hist. 66: 185 (1897).

Type: Ridley s.n. (K, lecto).

40. Phanera semibifida (Roxb.) Benth. var. acuminata (K. Larsen \& S.S. Larsen) Bandyop., P.P. Ghoshal et M.K. Pathak, comb. nov.

Bauhinia semibifida Roxb. var. acuminata K. Larsen \& S.S. Larsen, Nordic J. Bot. 13: 660 (1993). Type: F. Krispinus, SAN 105335 (K, image! holo; SAN, iso).

41. Phanera semibifida (Roxb.) Benth. var. bruneiana (K. Larsen \& S.S. Larsen) Bandyop., P.P. Ghoshal et M.K. Pathak, comb. nov.

Bauhinia semibifida Roxb. var. bruneiana K. Larsen \& S. S. Larsen, Nordic J. Bot. 13: 660 (1993). Type: J.P. van Neil 3455 (L, image! holo).

42. Phanera semibifida (Roxb.) Benth. var. longebracteata (K. Larsen \& S.S. Larsen) Bandyop., P.P. Ghoshal et M.K. Pathak, comb. nov.

Bauhinia semibifida Roxb. var. longebracteata K. Larsen \& S.S. Larsen, Nordic J. Bot. 13: 660 (1993). Type: Winkler 176 (HBG, holo; AAU, L, iso).

43. Phanera semibifida (Roxb.) Benth. var. perkinsiae (Merr.) Bandyop., P.P.Ghoshal et M.K.Pathak, comb. et stat. nov.

Bauhinia perkinsiae Merr., Publ. Bur. Sci. Gov. Lab. 17: 21 (1904), 'perkinsae'.

Bauhinia semibifida Roxb. var. perkinsiae (Merr.) K. Larsen \& S.S. Larsen, Nordic J. Bot. 13: 661 (1993). Type: E.D. Merrill 731 (NY, lecto; K, image! isolecto). 
44. Phanera stipularis (Korth.) Benth. var. brachystylus (K. Larsen \& S.S. Larsen) Bandyop., P.P. Ghoshal et M.K. Pathak, comb. nov.

Bauhinia stipularis Korth. var. brachystylus K. Larsen \& S.S. Larsen, Nordic J. Bot. 13: 659 (1993). Type: J. van Borssum Waalkes 2552 (L, image! holo; BO, K, iso).

45. Phanera steenisii (K. Larsen \& S. S. Larsen) Bandyop., P.P. Ghoshal et M.K. Pathak, comb. nov.

Bauhinia steenisii K. Larsen \& S.S. Larsen, Nordic J. Bot. 11: 630 (1991). Type: A. Kanis, SAN No. 50115 (L, image! holo; K, SAN, iso).

46. Phanera wrayi (Prain ex King) de Wit var. blumeana (K. Larsen \& S.S. Larsen) Bandyop., P.P. Ghoshal et M.K. Pathak, comb. nov.

Bauhinia wrayi Prain ex King var. blumeana K. Larsen \& S.S. Larsen, Nordic J. Bot. 13: 662 (1993). Type: T.C. Whitmore 3342 (K, holo; L, image! iso).

47. Phanera wrayi (Prain ex King) de Wit var. borneensis (K. Larsen \& S.S. Larsen) Bandyop., P.P.Ghoshal et M.K. Pathak, comb. nov.

Bauhinia wrayi Prain ex King var. borneensis K. Larsen \& S.S. Larsen, Nordic J. Bot. 13: 662 (1993). Type: W. Meijer 47492 (L, image! holo; K, SAN, iso).

48. Phanera wrayi (Prain ex King) de Wit var. cardiophylla (Merr.) Bandyop., P.P. Ghoshal et M.K. Pathak, comb. et stat. nov.

Bauhinia cardiophylla Merr., Philipp. J. Sci., C. 11: 79 (1916).

Bauhinia wrayi Prain ex King var. cardiophylla (Merr.) K. Larsen \& S.S. Larsen, Nordic J. Bot. 13: 662 (1993). Type: Native collector 1858 (A, image!). Dr. Emily W. Wood (pers. comm.) informed that there is only a photo of Native collector 1858 at A and not any specimen as cited by Larsen and Larsen (1996).

49. Phanera wrayi (Prain ex King) de Wit var. moultonii (Merr.) Bandyop., P.P. Ghoshal et M.K. Pathak, comb. et stat. nov.

Bauhinia moultonii Merr., Philipp. J. Sci., C. 11: 82 (1916).

Bauhinia wrayi Prain ex King var. moultonii (Merr.) K. Larsen \& S.S. Larsen, Nordic J. Bot. 13: 662 (1993). Type: Native collector, Bureau of Science no. 202 (A, holo; K, image! iso).

50. Phanera wuzhengyii (S.S. Larsen) Bandyop., P.P. Ghoshal et M.K. Pathak, comb. nov.

Bauhinia wuzhengyii S.S. Larsen, Novon 9: 526 (1999). Type: H.Y. Ying \& W. S. Kong 580812 (KUN, holo).

\section{Acknowledgements}

We are thankful to the Director, Botanical Survey of India, Kolkata and Additional Director, Central National Herbarium, Botanical Survey of India, Howrah for providing facilities; to Drs. A.M.S.F. Vaz, Instituto de Pesquisas Jardim Botanico do Rio de Janeiro, Brazil; A. Bruneau, Institut de recherche en biologie végétale, Université de Montréal, Canada and N. Malpure, Natural Products-Botany, Piramal Life Sciences Ltd., Mumbai, for providing literature. We are also thankful to the Directors/Curators/ Contact persons at A, C, FI, K, L, NY and P for providing us the images of the type specimens and to the anonymous reviewer for his helpful suggestions. 


\section{References}

Bandyopadhyay, S. 1999. Nomenclatural replacements in Bauhinia (Leguminosae: Caesalpinioideae). Kew Bull. 54: 974.

Bruneau, A., Mercure, M., Lewis, G.P. and Herendeen, P.S. 2008. Phylogenetic patterns and diversification in the caesalpinioid legumes. Botany 86: 697-718.

Larsen, K. and Larsen, S.S. 1996. Bauhinia. In: Kalkman, C., Kirkup, D.W., Nooteboom, H.P., Stevens, P.F. and Wilde, W.J.J.O. de (eds), Flora Malesiana 12: 442-535. Rijksherbarium/Hortus Botanicus, Leiden University, The Netherlands.

Lewis, G.P. and Forest, F. 2005. Cercideae. In: Lewis, G., Schrire, B., Mackinder, B. and Lock, M. (eds), Legumes of the World. Royal Botanic Gardens, Kew, pp. 57-67.

Queiroz, L.P. 2006. New species and new combinations in Phanera Lour. (Caesalpinioideae: Cercideae) from the Caatinga biome. Neodiversity 1: 6-10.

Sinou, C., Forest, F., Lewis, G.P. and Bruneau, A. 2009. The genus Bauhinia s.l. (Leguminosae): a phylogeny based on the plastid trnL-trnF region. Botany 87: 947-960.

Vaz, A.M.S.F. 2010. New combinations in Phanera (Leguminosae; Cercideae) from Brazil. Rodriguésia 61 (Suppl.): S33-S40.

Wunderlin, R.P. 2011. New combination in Phanera (Fabaceae). Phytoneuron 19: 1-2.

Wunderlin, R., Larsen, K. and Larsen, S.S. 1987. Reorganization of the Cercideae (Fabaceae: Caesalpinioideae). Biol. Skr. 28: 1-40. 